\title{
Effectiveness of Neuromotor Task Training for Children with Developmental Coordination Disorder: A Pilot Study
}

\author{
M.M. Schoemaker, A.S. Niemeijer, K. Reynders, B.C.M. Smits-Engelsman ${ }^{1}$ \\ Institute of Human Movement Sciences, University of Groningen, the Netherlands; \\ ${ }^{1}$ Nijmegen Institute for Cognition and Information, Nijmegen University, the Netherlands
}

\begin{abstract}
The aim of this pilot study was to evaluate the effectiveness of a Neuromotor Task Training (NTT), recently developed for the treatment of children with Developmental Coordination Disorder (DCD) by pediatric physical therapists in the Netherlands. NTT is a task-oriented treatment program based upon recent insights from motor control and motor learning research. Ten children with DCD (intervention group) were tested before and after 9 and 18 treatment sessions on the Movement $\mathrm{ABC}$ and a dysgraphia scale in order to measure the effectiveness of treatment on gross and fine motor skills in general and handwriting in particular. Five children (no-treatment control group) were tested twice with a time lag of nine weeks on the Movement $\mathrm{ABC}$ in order to measure spontaneous improvement. No improvement was measured for the children in the notreatment control group, whereas a significant improvement was found for children in the intervention group for both quality of handwriting and performance on the Movement $\mathrm{ABC}$ after 18 treatment sessions.
\end{abstract}

Reprint requests to: Dr. M.M. Schoemaker, Institute of Human Movement Sciences, University of Groningen, PO Box 196, 9700 AD Groningen, The Netherlands. e-mail: M.M.Schoemaker@PPSW.RUG.NL

\section{KEYWORDS}

clumsiness, physical therapy, handwriting, motor skills, Movement-ABC

\section{INTRODUCTION}

Over the past forty years, various treatment programs have been developed for children with Developmental Coordination Disorder (DCD). These treatment programs can roughly be divided into two categories: the so-called process-oriented approaches and the task-oriented approaches (Sugden \& Wright, 1998). The process-oriented approaches concentrate on the treatment of deficits in processes assumed to underlie poor motor coordination. Task-oriented approaches, on the other hand, focus directly at the functional skills with which a child experiences problems.

Examples of process-oriented approaches are kinesthetic training developed by Laszlo et al. (1988) and Sensory Integration Therapy developed by Ayres (1972). Laszlo et al. attributed a prominent role to kin aesthesis in the control of movement, and assumed that the motor co-ordination problems of children with DCD were the result of a deficit in kinesthetic awareness. Sensory Integration Therapy (S.I.T.) is based upon the assumption that children with learning disabilities in general and those with motor problems in particular are deficient in integrating perceptual information from various 
modalities. Treatment is directed at stimulating the tactile, vestibular, visual, and other sensory systems so that children learn to integrate sensory information into adequate (motor) responses. Among the physical and occupational therapists, S.I.T is one of the most popular approaches (Mandich et al., 2001).

Despite its popularity, however, not much evidence exists to suggest that S.I.T. or other process-oriented approaches are effective. Pless and Carlsson (2000) conducted a meta-analysis regarding the effectiveness of treatment approaches for DCD. The authors found a rather small mean effect size of 0.21 for process-oriented approaches. Moreover, in a review of evaluation studies, Mandich et al. (2001) found no support for the effectiveness of process-oriented approaches.

During the last decade, various researchers started to advocate a task-oriented approach to treatment. In Australia, Revie and Larkin (1993) developed a task-specific intervention program to increase motor competence by teaching functional motor skills. More recently, Missiuna and coworkers (2001) developed the 'Cognitive Orientation to daily Occupational Performance (CO-OP)' in Canada. In this program, the focus is on motor skill learning, but skill learning is enhanced by teaching problem solving techniques. Children learn to ask questions about their own performance and to find solutions for these questions. By applying these techniques, the authors aim to enhance transfer of motor learning outside the therapy program. Although the taskoriented approaches are relatively new, the first results regarding their effectiveness are promising. Pless and Carlsson (2000) found an effect size of 1.46 for these approaches in their meta-analysis.

In the Netherlands, a 'Neuromotor Task Training (NTT)' was recently developed for treating children with DCD by pediatric physical therapists (SmitsEngelsman et al., 2000). The training concerns a task-oriented program based upon recent insights about motor control and motor learning. Within this approach, physical therapists start with the assessment of the strengths and weaknesses of a child's functional performance. Next, therapists will analyze which cognitive or motor control processes might be involved in deficient motor skill performance. A child can fail to learn a specific motor skill because of attentional problems, fear of failure, lack of motivation, or lack of understanding how to execute a particular skill. In addition, motor-control processes might hamper successful performance, such as timing of the components of a motor skill pattern, motor planning, or parameter setting (the execution of a motor act with the required speed and force). In NTT, the functional exercises are designed in such a way that the therapist can analyze which motor control processes are deficient. For instance, if giving a secure and supportive surrounding improves ball catching, task training will aim at more psychological processes. If, however, a child can catch the ball only when standing still and warned beforehand, ball catching in complex and attentionally demanding situations will be trained. If the child has not yet developed a throwing pattern, the opportunity of merely throwing a variety of objects (size weight, material) will be given. Later a demand on parameterization will gradually be introduced by propelling the object over various distances or by aiming the objects at targets of different sizes. Through this approach, functional skills are trained in such a way that they tap the specific motor control processes that are thought to be involved.

Another important characteristic of NTT is that teaching principles derived from motor learning research are applied. Treatment approaches generally describe in detail the theories on which an approach is based, whereas information about the best way to instruct a particular treatment approach is discarded. The ultimate goal of treatment is not only to improve functional task performance 
during treatment but also to transfer learned skills to daily life performance. It is well known from research that motor learning in general and transfer in particular can be enhanced by applying the most effective motor learning principles. The literature research concerning motor learning has concentrated on three distinct subjects: (1) How to instruct people? (2) How to practice skills? (3) How to provide feedback?

It would be carrying it too far to summarize the results of research concerning motor principles in this paper. As it concerns the best way of instructing children, however, research findings indicate that motor learning in children may benefit from demonstrating a skill during the early phases of skill learning, whereas verbal instructions may be more helpful in later phases of skill learning (Magill, 1998). As it concerns practicing motor skills, research findings have emphasized the importance of practice variability, which refers to the variability in movements and context characteristics (as close as possible to real life situations) that a child encounters while practicing a skill (Magill, 1998). In addition, the provision of immediate or clear feedback may be essential for skill learning. Especially in children, motor learning may be enhanced by providing adequate knowledge of performance. Physical therapists trained in NTT learn to implement motor learning principles in clinical practice.

In this paper, the results of a pilot study are presented, aimed to investigate the effectiveness of NTT in a group of 10 children with DCD. A general motor test, the Movement $\mathrm{ABC}$ (Henderson \& Sugden, 1992), is used to evaluate improvement in both gross and fine motor skill performance. As children with DCD often experience problems with handwriting, which hinders academic functioning at school to a large extent, the effectiveness of NTT on a scale measuring dysgraphia is investigated as well.

\section{PILOT STUDY}

\section{Participants}

Fifteen children with DCD, nine boys and six girls (seven to ten years old), participated in this study. Ten children were included in the intervention group (seven boys and three girls; 7.1 to 9.2 years old). Five children were included in a no-treatment control group (two boys and three girls; 7.1 to 9.2 years old). All children in the intervention group were referred to a pediatric physical therapist by their general practitioner because of motor co-ordination problems. The children in the no-treatment control group were either referred to physical therapy $(n=3)$ or were recruited from schools because either their parents or teachers were concerned about their motor skills $(n=2)$. The inclusion criteria for both groups stipulated that: (1) all children obtained total scores on the Movement $\mathrm{ABC}$ below the $15^{\text {th }}$ percentile; (2) the motor problems of the children could not be attributed to evident pathological neurological signs; (3) only children attending schools for general education were included, which implies an IQ-score in the normal range.

The study was approved by the Medical Ethics Committee of the University Hospital of Groningen, the Netherlands.

\section{Assessment of motor function}

The Movement Assessment Battery for Children. The Movement ABC (Henderson \& Sugden, 1992) provides an indication of a child's motor functioning in daily life. Performance is related to motor norms using age-dependent standardized scores. The Movement $\mathrm{ABC}$ provides norms for children aged 4 to 12 years and the age norms are applicable to the Dutch population (SmitsEngelsman, 1998). Each of the four age-related 
batteries consists of eight items that measure different aspects of motor performance; three items measure manual dexterity, two items measure ball skills and three items are for static and dynamic balance. Children can score between 0 and 5 on each item, consequently total scores will vary from 0 to 40 , the higher the score the worse the performance. The total scores can be converted into percentile scores that show the child's level of performance in comparison with the child's peers. The test has acceptable validity and reliability (Henderson \& Hall, 1982; Lam \& Henderson, 1987). Inter-rater reliability for this test ranges from 0.70 to 0.89 , whereas test-retest reliability is 0.75 (Henderson \& Sugden, 1992).

The Concise Assessment Method for Children's Handwriting. The Concise Assessment Method for Children's Handwriting, or BHK (Hamstra-Bletz et al., 1987) is a screening tool for recognizing dysgraphia. The task consists of copying a standard text in 5 minutes or at least the first 5 lines if the child is a very slow writer. The standard text gradually increases in difficulty as the text proceeds. The first 5 sentences have a degree of difficulty equal to a grade 1 level reading exercise. The text is copied on unruled paper and is not read by the child before being copied.

Handwriting quality is evaluated using the dysgraphia scale of the BHK. This scale is based on the assessment of 13 dysgraphia features, namely, deviations from the standard hand or writing style. A score of 0 is given if the feature is absent. A maximum score for a feature is 5 . Each child's total score for all 13 items is then used to determine if the child is dysgraphic. The manual provides the following norms: (a) not dysgraphic: a score 0 to 21 ; (b) ambiguous: a score of 22 to 28 ; (c) dysgraphic: a score of 29 or higher.

The copying speed is determined by counting the number of letters written by the child in the first 5 minutes, including corrections. Satisfactory results are reported regarding inter-rater reliability on the items $(r=0.71$ to 0.89$)$ and intra-rater reliability for grades $2(r=0.87$ to $r=0.94)$ and 3 $(\mathrm{r}=0.79$ to $\mathrm{r}=0.88)$.

Procedure. All children were tested individually by a graduate student in human movement sciences trained in the administration of the tests. The student did not know in which group the children were included.

\section{Intervention}

Pediatric physical therapists treated the children in the intervention group individually for 30 minutes 18 times once a week. All therapists were qualified pediatric physical therapists who had received instruction in the principles of the NTT (see introduction). The content of the intervention was based upon the therapist's assessment of the child's motor performance on a range of tasks. Treatment goals were set for each child individually, which meant that the therapist treated those particular skills a child had found difficult during the assessment. The therapists were asked to record which skills and skill features were trained in each treatment session. Nine out of ten therapists complied with this request. One therapist refused because of lack of time. These records were used to determine whether children indeed did improve on the skills that were treated during therapy.

\section{Design}

Children in the intervention group were tested three times on the Movement-ABC and the BHK: before the start of intervention (T1), after 9 intervention sessions (T2), and after another 9 intervention sessions (T3). Intervention was provided for 30 minutes once a week. Children in the no-treatment control group were tested twice ( $\mathrm{T} 1$ and $\mathrm{T} 2$ ) with a period of 9 weeks without any intervention in between to measure spontaneous 
improvement. As 3 of the 5 children in the notreatment control group were on a waiting list for physical therapy, withholding intervention any longer to measure the spontaneous improvement during another 9 weeks was not ethical. For this group, only the data from the Movement-ABC were available for comparison of $\mathrm{T} 1$ and $\mathrm{T} 2$. Children in the no-treatment control group were not tested on the dysgraphia scale.

\section{Statistics}

As the data were not normally distributed, the Wilcoxon Signed Ranks Test was applied to test for the differences between test moments for each group separately.

\section{RESULTS}

\section{Movement ABC}

In Fig. 1, the change in median scores for the intervention and the no-treatment control groups across measuring moments is presented. At T1, no significant difference was found between the two groups $(p=0.07)$. In addition, no significant change was found between $\mathrm{T} 1$ and $\mathrm{T} 2$ for the notreatment control group $(p=0.49)$. For the intervention group, no significant improvement was found between $\mathrm{T} 1$ and $\mathrm{T} 2(\mathrm{p}=0.33)$. The intervention group, however, significantly improved from $\mathrm{T} 1$ to $\mathrm{T} 3(\mathrm{p}=0.018)$. When examining the subtests of the Movement-ABC, we found a significant improvement between $\mathrm{T} 1$ and $\mathrm{T} 3$ for manual dexterity and ball skills $(p=0.028$ and $p=0.018$ respectively), but not for balance $(p=0.108)$. In Table 1 , the distribution of children among the different categories of the Movement$\mathrm{ABC}$ is presented for the intervention group at $\mathrm{T} 1$ and T3. In Table 2, the number of children who

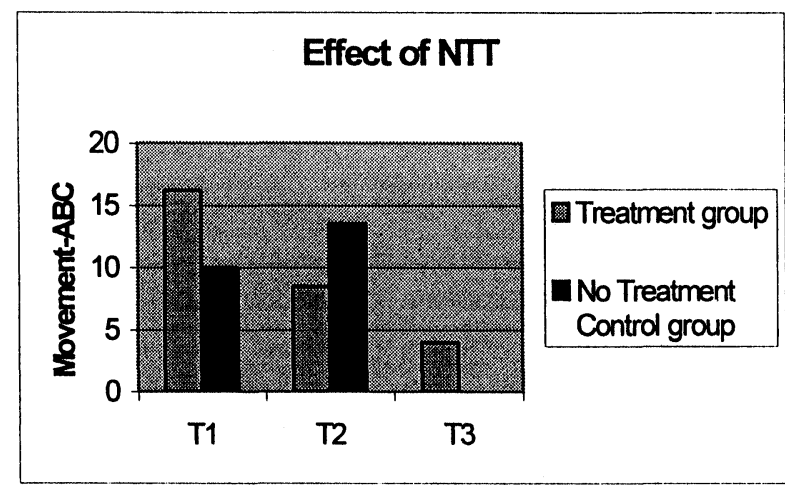

Fig. 1: Median total scores on the Movement-ABC for the treatment group and the no-treatment control group at each test moment.

\section{TABLE 1}

Performance of children in the intervention group in the Movement- $\mathrm{ABC}$ before and after treatment

\begin{tabular}{|c|c|c|c|}
\hline \multirow{2}{*}{$\begin{array}{c}\text { Time of } \\
\text { test }\end{array}$} & \multicolumn{3}{|c|}{ Group/percentile } \\
\cline { 2 - 4 } & Normal & At risk & Deviant \\
\cline { 2 - 4 } & $15^{\text {th }}-100^{\text {th }}$ & $5^{\text {th }}-15^{\text {th }}$ & $0-5^{\text {th }} \%$ \\
\hline T1 & - & 3 & 7 \\
\hline T3 & 7 & 2 & 1 \\
\hline
\end{tabular}

$\mathrm{T} 1=$ before treatment $; \mathrm{T} 3=$ after treatment

improved, stayed at the same performance level, or whose performance worsened are presented for each item of the Movement-ABC. An improvement is a change in score of at least 1 point.

\section{BHK}

In Table 3, the median scores for handwriting quality and handwriting speed (number of letters written in five minutes) are listed for the intervention group. A significant improvement was found for handwriting quality between $\mathrm{T} 1$ and $\mathrm{T} 3$ $(p=.017)$, but not for handwriting speed $(p=0.11)$. 
TABLE 2

Performance of children in each item of the Movement-ABC

\begin{tabular}{llccc} 
& \multicolumn{1}{c}{ Items of the Movement ABC } & \multicolumn{2}{c}{ Number of children } \\
\hline Item no. & \multicolumn{1}{c}{ Task } & Improved & Not improved & Decreased \\
\hline Item 1 & Speed and accuracy of each hand separately & 5 & 5 & - \\
\hline Item 2: & Coordination of two hand performing a single operation & 1 & 8 & 1 \\
\hline Item 3 & Hand-eye coordination in pencil control & 4 & 5 & 1 \\
\hline Item 4: & The ability to accurately propel an object & 6 & 3 & 1 \\
\hline Item 5: & The ability to catch a moving object & 9 & - & 1 \\
\hline Item 6: & Static balance & 5 & 3 & 2 \\
\hline Item 7: & Dynamic balance during fast and explosive movements & 3 & 7 & - \\
\hline Item 8: & Dynamic balance during slow, controlled movements & 6 & 3 \\
\hline
\end{tabular}

\section{TABLE 3}

Median scores on the handwriting assessment scale (BHK) for the intervention group before and after 18 sessions of physical therapy.

\begin{tabular}{|l|c|c|}
\hline Intervention group: & $\mathrm{T} 1$ & $\mathrm{~T} 3$ \\
\hline Handwriting-Quality & 34 & 24.5 \\
\hline Handwriting-Speed & 106 & 118.5 \\
\hline
\end{tabular}

$\mathrm{T} 1=$ before treatment; $\mathrm{T} 3=$ after treatment

Table 4 is an overview of the classification of each child in the different diagnostic categories of the BHK-quality scale before and after treatment. Table 5 shows an overview of the number of children who did or did not improve on handwriting, manual dexterity, ball skills, and balance and whether these aspects were practiced
TABLE 4

Performance of children in the intervention group on the handwriting assessment scale (BHK) before treatment and after 18 sessions of physical therapy*

\begin{tabular}{|l|c|c|}
\hline Handwriting-Quality: & $\mathrm{T} 1$ & $\mathrm{~T} 3$ \\
\hline Not dysgraphic & - & 10 \\
\hline Ambiguous & 2 & 5 \\
\hline Dysgraphic & 7 & 3 \\
\hline
\end{tabular}

$\mathrm{T} 1$ = before treatment; $\mathrm{T} 3=$ after treatment

*The data from one child are missing because the BHK was not administered at one test moment.

during treatment. From this table it becomes clear that children generally did improve when a particular skill was practiced and did not improve when these skills were not specifically practiced. Only balance forms an exception, even when 
TABLE 5

Effect of practice on the improvement of children in a particular aspect of motor functioning.*

\begin{tabular}{|l|c|c|}
\hline & Improved & $\begin{array}{c}\text { Not } \\
\text { improved }\end{array}$ \\
\hline Handwriting-quality & & 1 \\
Practiced & 4 & 2 \\
Not-practiced & 1 & - \\
\hline Manual Dexterity & 4 & 4 \\
Practiced & 1 & - \\
Not-practiced & 7 & 1 \\
\hline Ball skills & 1 & 3 \\
Practiced & & 1 \\
Not-practiced & 3 & 2 \\
\hline Balance & & \\
Practiced & & \\
Not-practiced &
\end{tabular}

*Data regarding handwriting quality from one child are missing because the BHK was not administered at one test moment.

therapists did not mention balance as a treatment goal, some children still improved on the balance tasks of the Movement-ABC.

\section{DISCUSSION}

The aim of this study was to evaluate the effectiveness of a recently developed intervention program (NTT) for children with DCD. Although a relatively small group of children was included, in concordance with results found in other studies, a positive effect of this task-oriented approach was found. Children with DCD improved on both gross and fine motor skills measured by the Movement$\mathrm{ABC}$ after 18 treatments with NTT, whereas the no-treatment control group did not improve at all during 9 weeks without intervention. As can be seen in Fig. 1, the median scores of the notreatment control group even tended to deteriorate (although not significantly) during the 9 weeks without intervention. This result is in contrast to that in the intervention group, who obtained better scores after 9 weeks of intervention. That such improvement did not yet reach the set significance level might be due to the small sample size. Inspection of the individual results of the intervention group revealed that all children improved their scores on the Movement-ABC, and nine children to such an extent that they could be classified in a different performance category on this test. A similar improvement was found for handwriting quality.

At present, we may conclude that the first results regarding the effectiveness of NTT are promising. Because the sample size was small, however, further investigation of the effectiveness of NTT is warranted, despite the consistent improvements across children. The results concern the first data of a larger study in which we aim to include at least 50 children with DCD. In addition, the different selection criteria prevailing in the intervention group and the no-treatment control group resulted in different (although non significant $(p=0.07))$ median scores on the Movement-ABC at the pre-test. Although not likely, whether the difference in pre-test scores influenced the susceptibility to the NTT treatment in this pilot study is unknown. Lastly, although NTT proved effective in this pilot study, we do not yet know whether the effects are larger than those of more traditional intervention approaches. Studies are asked for in which the effects of NTT are compared with the effects of traditional intervention approaches, such as S.I.T.

One might argue that the improvement noticeable in both instruments used to evaluate the effectiveness of intervention pleads against a taskoriented effect, but rather reflects a processoriented effect. In a task-oriented approach, specific 
effects of treatment are expected in practiced tasks, whereas no effect of treatment should be visible in non-practiced tasks. When a general improvement is observed across tasks, seemingly a deficit in an underlying process is influenced by intervention. Yet, the data regarding the number of children who improved on each item of the Movement-ABC pleads against this argument. Definitely not all children improved on all items of this test. Large improvements were visible for such tasks as catching a moving object, accurate propulsion of an object, the speed of one-hand coordination, and dynamic balance during slow movements, whereas hardly any improvement was visible for bi-manual tasks or dynamic balance during fast movements. In addition, when we examined the relation between the kind of motor skills that were practiced during treatment and the improvement on those particular skills, the specific improvement of children with DCD on those aspects that were practiced became clear. If ball skills, manual dexterity, or handwriting was practiced, such children improved on these skills, but they did not improve when these skills were not practiced. Only balance was an exception in this respect. Even when balance tasks were not specifically practiced during treatment, some children with DCD did improve on the subtest balance of the Movement-ABC. It might be that while practicing other skills, postural control was indirectly treated as well, as postural control is essential for the execution of many motor skills and was thus implicitly trained. Consequently, these children demonstrated better postural control during the balance tasks of the M-ABC than did those without intervention.

A more curious finding regarding balance, however, is that three children obtained worse scores on balance after intervention, even though balance was specifically practiced during intervention. One possible explanation might be that balance is a rather broad category. For instance, static balance might be practiced during treatment, but dynamic balance not. As a consequence children might obtain better scores on static balance, but worse scores on the not-practiced dynamic balance items of the Movement-ABC. As static balance is measured only in one item of the test, and dynamic balance in two items, the net results might be a worse score on balance as a whole, although improvement did take place.

The question is how to explain the positive results of task-oriented approaches in contrast to process-oriented approaches? The effects of taskoriented training might be explained by a recent theory about neural development, the Neuronal Group Selection Theory (NGST; Sporns \& Edelman, 1993). According to this theory, during development neuronal groups or networks are formed that consist of large amounts of strongly interconnected neurons. The structure and function of such networks are selected by develop-ment, behavior, and contextual factors. Each child is born with a primary neuronal repertoire. Such a repertoire, determined by evolution, consists of multiple neuronal groups. During development, selection occurs as a consequence of behavior and experience. As a consequence, behavior becomes less variable. The variability soon returns, however, due to the enormous amount of information to which a child is exposed. As a result, the connectivity within neuronal groups changes (secondary repertoire), which allows for situation specific motor behavior (secondary or adaptive variability). According to Hadders-Algra (2000), children with DCD can show deficits in secondary variability, which means that they are not able to adapt their motor behavior to the specific demands of the situation. In her opinion, intervention should provide active practice in the skills that are deficient to enhance the right selection of neuronal groups that will lead to an increment of adaptation of motor behavior. Also Ulrich (2000) states that patterns of movement that are repeated frequently 
generate strong neural pathways that support the movement pattern. Therefore, task-oriented interventions like NTT, which concentrate on the active practicing of the skills that are problematic for a child, can increase secondary or adaptive variability. Our pilot study demonstrates that such intervention can lead to improvement of motor skills in children with DCD. The results, however, have to be interpreted with caution considering the small size of the groups and that the no-treatment control group could be followed for only 9 weeks.

\section{REFERENCES}

Ayres AJ. 1972. Sensory Integration and learning disorders. Los Angeles, California, USA: Western Psychological Services; 294.

Hadders-Algra M. 2000. The neuronal group selection theory: promising principles for understanding and treating developmental motor disorders. Dev Med Child Neurol 42:707-715.

Hamstra-Bletz E, Blöte AW. 1993. A longitudinal study on dysgraphic handwriting in primary schools. J Learn Disabil 23: 689-699.

Henderson SE, Hall DMB. 1982. Concomitants of clumsiness in young school children. Dev Med Child Neurol 24: 448-460.

Henderson SE, Sugden DA. 1992. The Movement Assessment Battery for Children. San Antonio, Texas, USA: The Psychological Corporation; 240.

Lam J, Henderson SE. 1987. Some applications of the Henderson revision of the Test of Motor Imparment. Br J Educ Psychol 57: 389-400.

Laszlo JI, Bairstow PJ, Bartrip J, Rolfe VT. 1988. Clumsiness or perceptuo-motor dysfunction. In: Colley A, Beech J, eds, Cognition and action in skilled behaviour. Amsterdam, the Netherlands: North Holland: 293-316.

Magill RA. 1998. Motor learning: concepts and applications. Boston, Massachusetts, USA: McGraw-Hill; 326.

Mandich AD, Polatajko HJ, Macnab JI, Miller LT. 2001. Treatment of children with Developmental Coordination Disorder: what is the evidence? Phys Occup Ther Pediatr 20: 51-68.

Missiuna C, Mandich AD, Polatajko HJ, MalloyMiller T. 2001. Cognitive Orientation to Daily Occupational Performance (CO-OP): Part 1Theoretical Foundations. Phys Occup Ther Pediatr 20: 69-82.

Pless M, Carlsson M. 2000. Effects of motor skill intervention on Developmental Coordination Disorder: A Meta-analysis. Adapt Phys Act Quart 17: 381-401.

Revie G, Larkin D. 1993. Task-specific intervention with children reduces movement problems. Adapt Phys Act Quart 10: 29-41.

Smits-Engelsman BCM 1998. Movement ABC; Nederlandse Handleiding (Dutch Manual). Lisse, the Netherlands: Swets and Zeitlinger; 128.

Smits-Engelsman BCM, Reynders K, Schoemaker MM. 2000. Kinderen met Developmental Coordination Disorder: Symptomatologie, diagnostiek en behandeling. In: Van Empelen R, Nijhuis R, Hartman, A, eds, Kinderfysiotherapie. Maarssen, the Netherlands: Elsevier; 505-522.

Sporns O, Edelman GM. 1993. Solving Bernstein's problem: a proposal for the development of coordinated movement by selection. Child Dev 64: 960-981.

Sugden DA, Wright HC. 1998. Motor coordination disorders in children. London, England: Sage Publications; 131.

Ulrich D. 2000. Test of Gross Motor Development, Second Edition. Austin, Texas, USA; Pro-Ed; 60. 

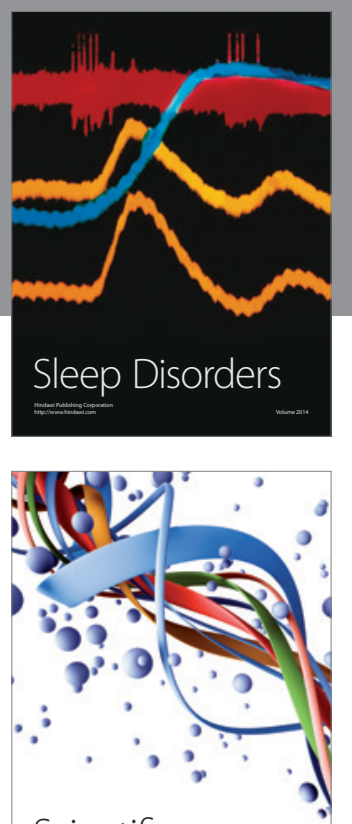

Scientifica
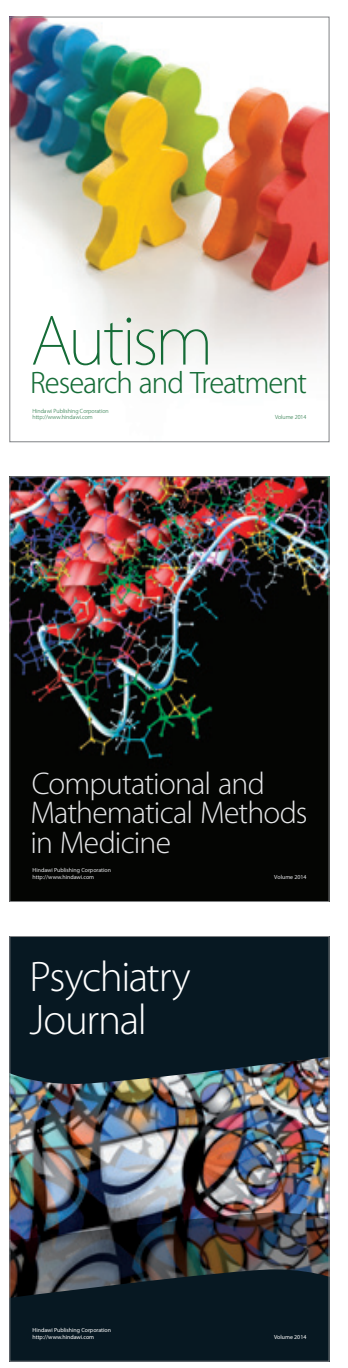
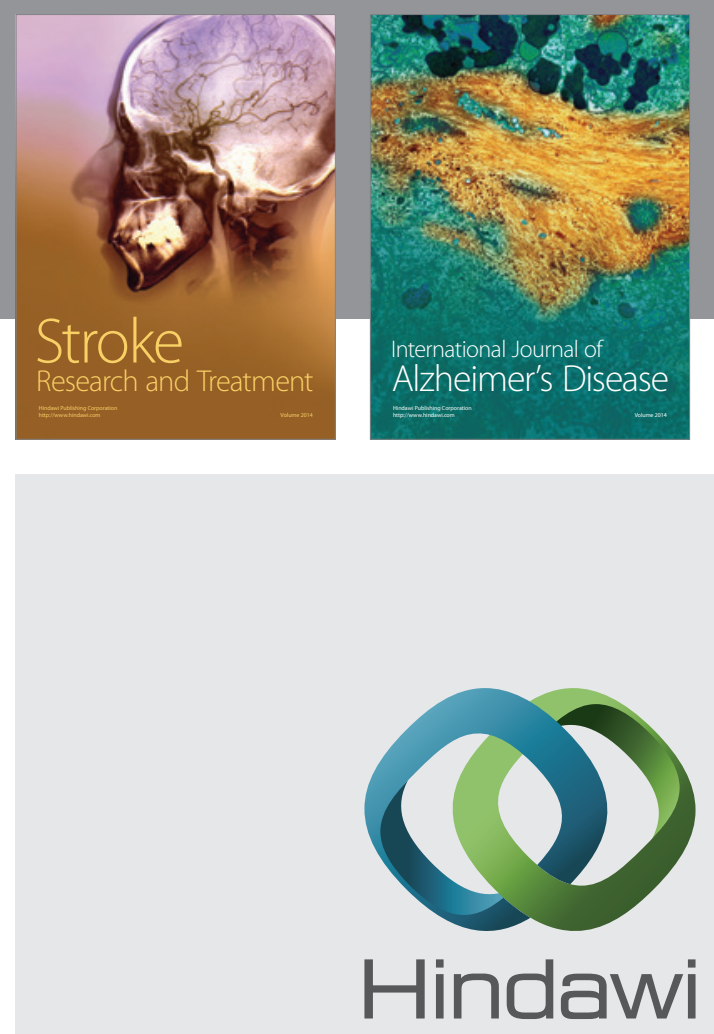

Submit your manuscripts at

http://www.hindawi.com
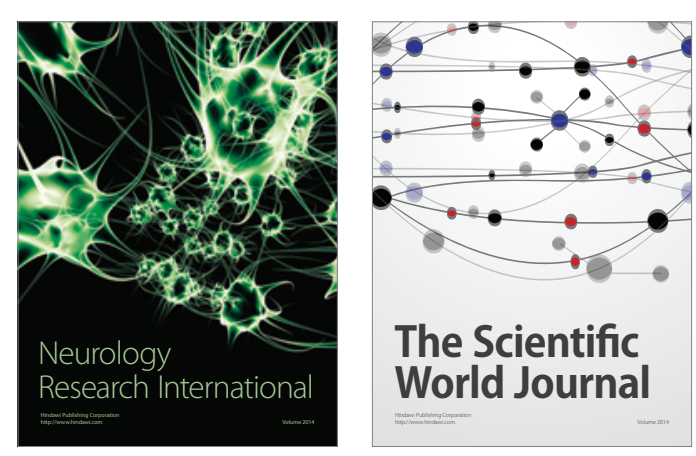

The Scientific World Journal

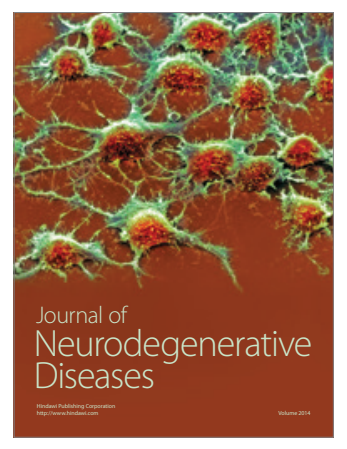

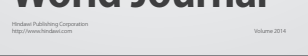

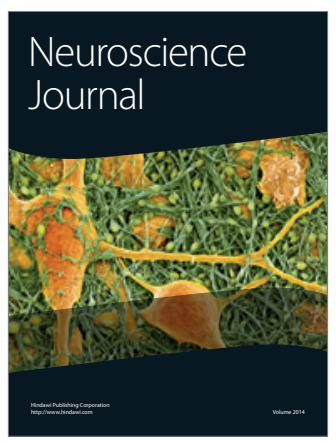

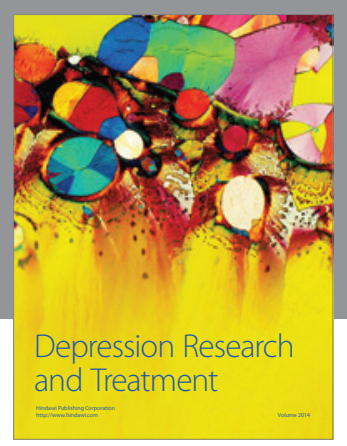
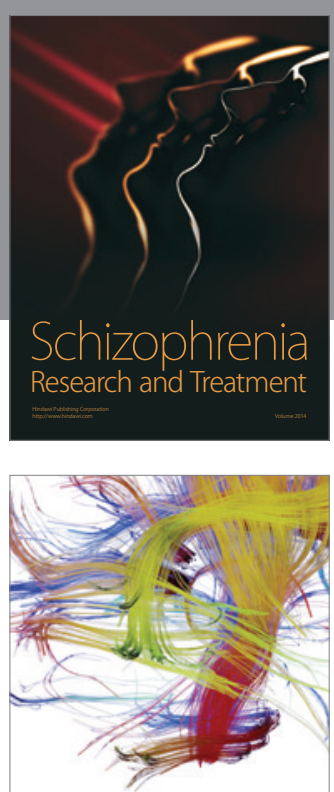

Brain Science

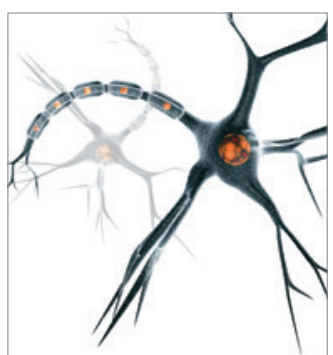

Neural Plasticity
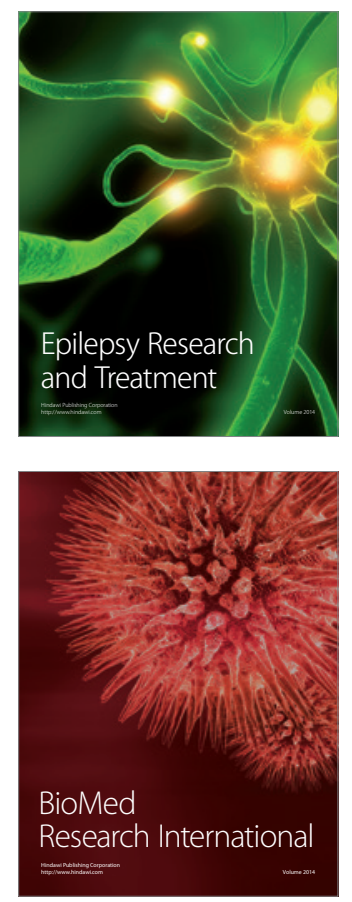

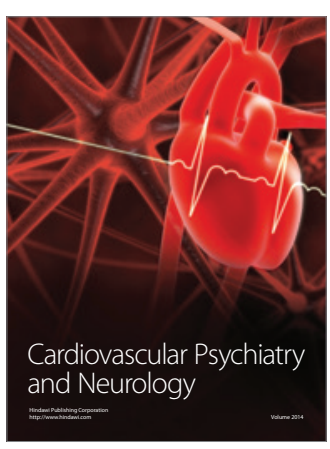

Parkinson's

Disease
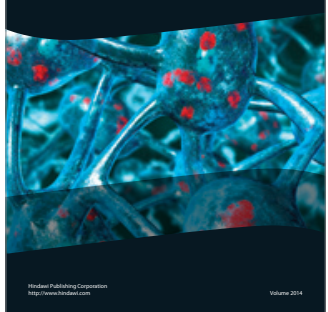